\title{
Experiment on the Constancy of the Velocity of Electromagnetic Radiation
}

\author{
Petr Beckmann ${ }^{1}$ and Peter Mandics \\ Contribution From Department of Electrical Engineering, University of Colorado, Boulder, Colo.
}

(Received July 22, 1964)

\begin{abstract}
An experiment by Kantor, reporting results in sharp contradiction to Einstein's Second Postulate, was repeated using the coherent light of a laser. The results were found to be consistent with the Special Theory of Relativity. It is concluded that the ballistic hypothesis of light propagation disregarding the effect of air is incorrect. It is pointed out that the direct experimental evidence in favor of Einstein's Second Postulate is surprisingly meager and further experiments to confirm or reject the ballistic hypothesis are envisaged.
\end{abstract}

\section{Introduction}

The results of an experiment reported by Kantor [1962] sharply contradicted Einstein's postulate of the constant velocity of light and seemed to give strong support to the "ballistic" theory of light, according to which the velocity of light is

$$
\mathbf{c}=\mathbf{c}_{0}+\mathbf{v}
$$

where $\mathbf{v}$ is the velocity of the source with respect to the observer and $c_{0}=3 \times 10^{8} \mathrm{~m} / \mathrm{sec}$ is the velocity of light with respect to its source; this theory will explain most direct experiments if one regards reflecting or transparent objects as secondary sources reradiating the incident light with a velocity given by the same formula and independent of the velocity of the incident light. Kantor's results also indicated that the air would not, over short distances, significantly decelerate light by reradiation.

An analysis undertaken by one of us [Beckmann 1963] shows that Kantor's result is not as impossible as one might, at first sight, conclude in view of the successes and correct predictions of the Special Theory of Relativity in elementary-particle physics. The experiment was therefore repeated (in air) with two modifications: coherent light of a laser was used in the experiment, and automatic synchronization was achieved by a chopping mirror. Our result contradicts Kantor's observations and is consistent with Einstein's postulate in general and the findings of Babcock and Bergman [1964] in particular.

\footnotetext{
1 On leave from Institute of Radio Engineering and Electronics, Czechoslovak Academy of Sciences, Prague 8, Czechoslovakia.
}

\section{Necessity of Checking Einstein's Second Postulate}

The reason why Kantor's results and interpretation might indeed have been correct and could not simply be dismissed as a single report in the face of overwhelming evidence to the contrary is, briefly, the following:

A single and unfinished attempt to found new electrodynamics [Ritz, 1908] which would both comply with the Galilei principle of relativity (as distinct from Lorentz-relativity) and also agree with all experimental measurements has failed, but there is no a priori reason to believe that such an attempt must always be doomed to failure; it is therefore incorrect to invoke the successes of the Einstein theory, as far as they are directly or indirectly based on our present Maxwell-Lorentz electrodynamics, as proof of the Second Postulate: it could still be that the Lorentz transformation is only the right equivalence-formula correcting inaccurate electrodynamics (for high velocities) by adequately deforming space and time. An experiment to confirm or reject this possibility must therefore test the constancy of the speed of light by direct measurement and without inferences based on our present electromagnetic theory. This requirement rules out practically all elementary-particle experiments. From another point of view, it has already been pointed out by Fox [1962] and Dingle [1960a, b, c] that neither double stars nor high-energy particles give any clear-cut evidence in favor of the Second Postulate. Also, some theoretical difficulties in the Einstein theory have been discovered; one of the most perplexing paradoxes (that seems to have gone unanswered) is the velocity of propagation of a Doppler effect 
discovered by Dingle [1960b]. The direct laboratory experiments performed with light to settle the question of a dependence of the velocity of light on that of its source ['Tolman, 1910, 1912; Majorana, 1917, 1918a, 1918b, 1919; Tomaschek, 1924; BonchBruyevich and Molchanov, 1956] do not contradict the above type of a ballistic theory, for the intent of these experiments is always thwarted by the presence of a beam splitter of other glass object, which according to the hypothesis under consideration would act as a secondary source and reradiate the incident light, decelerating it to the velocity $c_{0}$. These experiments are therefore irrelevant. ${ }^{2}$ The one exception is Michelson's experiment with rotating mirrors [1913]; however, for this experiment the hypothesis under consideration (which Michelson failed to consider fully) leads to a result not well outside the experimental error. That the conclusions drawn from the above experiments went unchallenged for half a century is easily understood when it is remembered that the question of a possible dependence of the velocity of light on that of its source was never under dispute in the great controversy between the ether and relativity theories, both theories denying any such dependence. ${ }^{3}$

From the above points of view the evidence contradicting Kantor's result, far from being overwhelming, was thus found to be very meager and it was therefore considered important to repeat the experiment.

\section{Experimental Setup and Measurements}

The experimental setup is shown schematically in figure 1. The beam from a helium-neon laser (Spectra-Physics Model 130, $6328 \AA$ A, 0.2 to $0.5 \mathrm{~mW}$, diameter of beam $2.5 \mathrm{~mm}$, divergence $80 \mathrm{sec}$ of arc) is reflected from the chopping mirror (CM) and the

2 In the Ives-Stilwell type of experiment the velocity of the ions is not measured directly, but inferred from electromagnetic theory (the Lorentz force law). ${ }^{3}$ We consider the ether theory thoroughly disproved and disregard it throughout this paper.

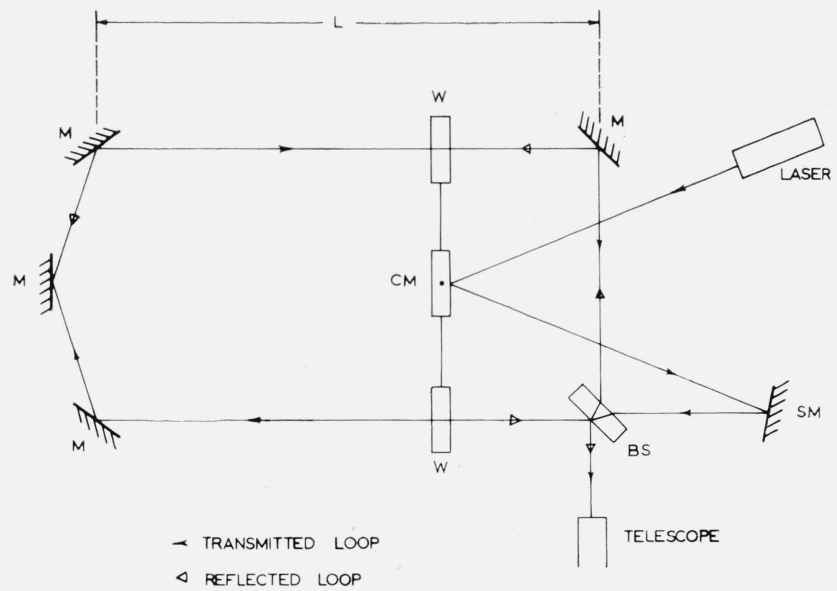

Figure 1. Schematic drawing of the experimental setup. stationary mirror (SM) into the beam splitter (BS), where it is split into a reflected and a transmitted beam. Both beams traverse the loop of a Fizeau interferometer [Born and Wolf, 1959, p. 301] in opposite directions, are reunited by the beam splitter and form interference fringes localized at infinity, which may be observed through a telescope. Both beams passed through glass windows $(\mathrm{W})$ of thickness $0.15 \mathrm{~mm}$. The windows $(1 \mathrm{~cm}$ by $1 \mathrm{~cm})$ were mounted on a rotor, at equal distances from its axis of rotation, their centers $24.4 \mathrm{~cm}$ apart. The rotor was rotated by an electric motor, the speed of which could be varied and reversed. The chopping mirror was also mounted firmly to the rotor so that the interferometer was in action only at the moment when the windows were in the position shown in figure 1 ; if the rotor was off the perpendicular position by more than $0.5^{\circ}$, the chopping mirror diverted the incoming ray from its path into the interferometer and nothing could be seen in the telescope. The interferometer was alined by adjusting the position of the beam splitter and the mirrors (M). It was first adjusted in collimated white light by making the two images of a pin mounted in front of the source coincide; similarly, the two images of the laser spot (projected through the telescope onto a screen) were made to coincide. In addition, the actual coincidence of the reflected and transmitted loops could easily be checked, owing to the small diameter of the laser beam, at any point along the loop of the interferometer by inserting a piece of translucent paper and alternately interrupting the reflected and transmitted beams: the two spots were coincident on the paper. In this way a zero fringe (uniform illumination throughout the field of view) was obtained and the beam splitter was then very slightly rotated about its vertical axis, thus very slightly displacing the reflected from the transmitted loop and giving rise to low-order fringes. By progressively covering up each of the windows with a sheet of paper, it was verified that both beams passed through both windows; this was also confirmed by observing the reflection on the rotating windows in the dark. The coherence of the laser light introduces some additional fringes; e.g., by interference of the light reflected from the front and back surfaces of the beam splitter (which is, of course, too thick to produce interference fringes in incoherent light). These unwanted fringes were suppressed by screening off the unwanted rays (fig. 2). Figure 3 shows the entire setup and figure 4, a detail of the rotor with the chopping mirror and the windows.

If now the rotor is rotated so that the windows attain a circumferential velocity which, during the pertinent fraction of a degree of rotation, is for all practical purposes uniform along the beams of light passing through them, the Einstein theory predicts no fringe shift (except one due to the dragging coefficient in the windows; this is easily shown negligible and unobservable), whereas the ballistic hypothesis in its reradiation version predicts a fringe shift, since the windows would accelerate the light traveling around the loop in the direction of rotation of the 


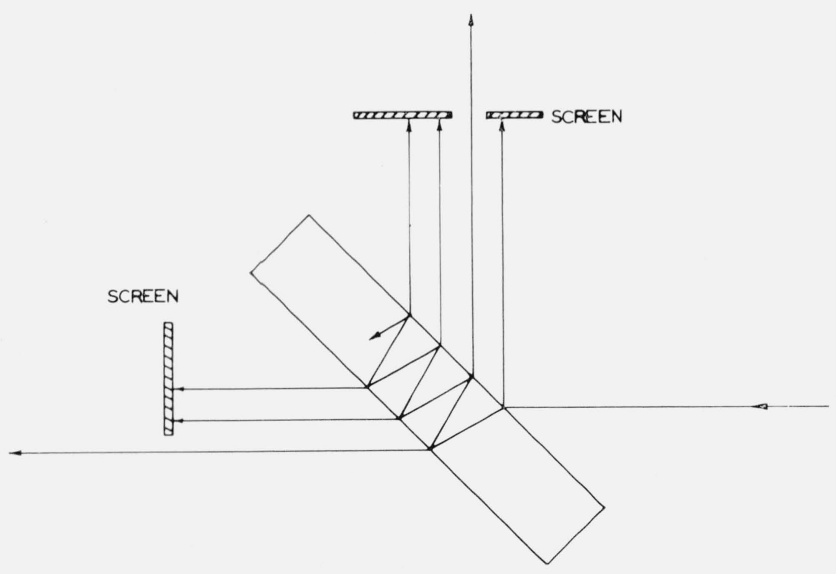

Figure 2. Multiple reflections by the beam splitter.

Interference between the indicated rays caused additional fringes, which were eliminated by screening off the unwanted rays as shown. A double-layer beam eliminated by screening off the unwanted rays as shown. A double-layer beam splitter was used, so that the
than indicated in the figure.

windows and decelerate it for the loop in the opposite direction. The corresponding calculation [Kantor, 1962] yields a relative fringe shift

$$
\Delta=\frac{2 \beta L}{\lambda}
$$

with respect to the fringes when the windows are stationary. On reversing the motor the shift should therefore be double this amount, i.e., $4 \beta L / \lambda$. In this formula, $L$ is the length of the interferometer (fig. 1), $\lambda$ the wavelength of the light and $\beta=v / c_{0}$, where $v$ is the circumferential velocity of the windows.

In our case $L$ was $1.572 \mathrm{~m}, \lambda=6.328 \times 10^{-7} \mathrm{~m}$, and most measurements were taken at a speed of 2100 $\mathrm{rpm}$ of the motor, making $\beta=8.94 \times 10^{-8}$. On reversing the motor, the relative shift predicted by the ballistic hypothesis, neglecting the effect of the air, is therefore 0.900 of a fringe. A shift of this magnitude would of course readily be detected in the telescope, where it would show against the crosshair of the telescope. Observations were made as follows: The fringe pattern was observed relative to the telescope crosshair with the motor running at a certain speed (measured by illuminating the rotor by a Strobo-Tac). The motor was then reversed by suddenly reversing the field current. The fringe pattern relative to the crosshair was then continuously observed while the motor was slowing down, gathering speed in the opposite direction and reaching a steady speed (which differed only very slightly from the one in the opposite direction). It is estimated that a shift of about 0.1 of a fringe would have been detected; but in fact no shift was observed. Figure 5 shows a typical photograph of the fringes for +2100 and $-2100 \mathrm{rpm}$ of the motor.

When the beam passed through the windows near their edges (the entire assembly of the motor including the rotor with the windows could be raised and

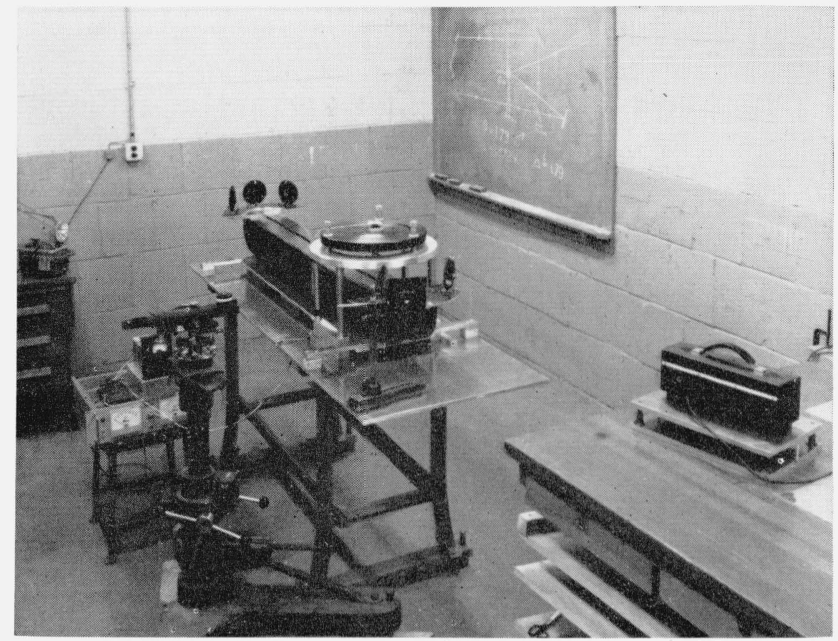

Figure 3. View of the experimental setup.

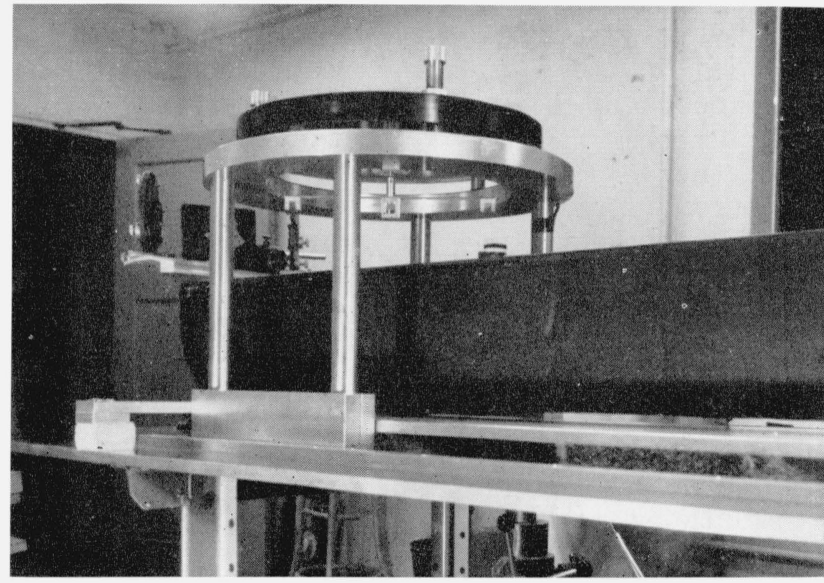

Figure 4. Rotor with chopping mirror and windows.

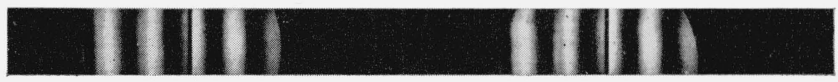

Figure 5. Example of fringes at $+2,100$ and $-2,100 \mathrm{rpm}$ of the motor.

Photographs taken with a Polaroid camera, 3000 ASA film.

lowered with respect to the interferometer, cf. fig. 4), the bottom part of the fringes would curve. This distortion of the fringes increased with velocity and might easily be mistaken for a shift; that this "shift" was not, however, due to a change in the velocity of light (but evidently due to mechanical deformations of the rotating windows and possibly also to the turbulent and compressed air in their vicinity) was shown by reversing the motor - the "shift" did not change direction. 


\section{Conclusions}

Our result is in agreement with that of Babcock and Bergman [1964] and contradicts that of Kantor [1962]. It is also consistent with the Special Theory of Relativity.

We conclude that the ballistic hypothesis in the reradiation version with air at atmospheric pressure having no substantial effect (i.e., not completely preventing a possible change in the velocity of light) is incorrect.

The next step to check the possible validity of the ballistic hypothesis is to repeat the experiment in vacuum, thus removing objections that the air might decelerate the light. This has already been done by Babcock and Bergman [1964], and also by Rotz [1963], who used a three-slit interferometer with one of the slits moving. Their results are again negative. However, to make such experiments completely conclusive, the interferometer path should be as free of air molecules as possible. One might, for example, require that the great majority of photons traveling along the interferometer will not collide with air molecules. Calculations [Beckmann and Mandies, 1965] show that the vacuum used by Babcock and Bergman $\left(10^{-2}\right.$ torr $)$ or Rotz $\left(10^{-4}\right.$ torr) was not high enough to meet this requirement; the necessary vacuum is of the order of $10^{-10}$ torr.

It was therefore decided to perform a further test of the ballistic hypothesis in a much higher vacuum. The interferometer described above was not used in this experiment, as it may cause a fringe shift by mechanical deformation of the rotor and the windows at high speeds. The test used a Lloyd mirror with the moving parts outside the interferometer, thus precluding a shift due to mechanical deformations. This experiment, performed in a vacuum better than $10^{-6}$ torr, once more yielded a negative result as will be reported elsewhere [Beckmann and Mandies, 1965].

The above investigation was made possible by a grant of the Office of Research and Creative Activities of the University of Colorado. Every possible assistance was given to us by Dr. Frank S. Barnes. Some of the optical and measuring equipment was kindly lent to us by the U.S. Navy Electronics Laboratory, San Diego, Calif., the National Bureau of Standards, Boulder, Colo., and the High Altitude Observatory of the University of Colorado. Justin
B. Pierce was most cooperative in machining the parts. W. Kantor designed some of the mechanical parts of the apparatus. We are also grateful to Mrs. Charlotte Cranford, who typed the manuscript.

\section{References}

Babcock, G. C., and T. G. Bergman (1964), Determination of the constancy of the speed of light, J. Opt. Soc. Am. 54, 147-151.

Beckmann, P. (May 1963), A re-examination of the experiments on the velocity of light with moving sources, URECSAV Rept. No. 30.

Beckmann, P., and P. Mandics (1965), A test of the constancy of the velocity of electromagnetic radiation in high vacuum (to be published).

Bonch-Bruvevich, M. A., and V. A. Molchanov (1956), A new optical relativistic experiment (in Russian), Opt. i Spektroskopiya 1, 113-124.

Born, M., and E. Wolf (1959), Principles of optics (Pergamon Press, London-New York-Oxford-Paris).

Dingle, H. (1960a), Relativity and electromagnetism: an epistemological appraisal, Phil. Sc. 27, 233-253.

Dingle, H. (1960b), The Doppler effect and the foundations of physics, Br. J. Phil. Sc. 11, 11-31 and 113-129.

Dingle, H. (1960c), The origin and present status of the special relativity theory, Science Progress 48, 201-219.

Fox, J. G. (1962), Experimental evidence for the second postulate of relativity, Am. J. Phys. 30, 297-300.

Kantor, W. (1962), Direct first-order experiment on the propagation of light from a moving source, J. Opt. Soc. Am. 52, 978-984.

Majorana, Q. (1917), Démonstration expérimentale de la constance de vitesse de la lumière réflechie par un miroir en mouvement, Comp. Rend. Ac. Fr. 165, 424-426.

Majorana, Q. (1918a), Démonstration expérimentale de la constance de vitesse de la lumière émise par une source mobile, Comp. Rend. Ac. Fr. 167, 71-73.

Majorana, Q. (1918b), On the second postulate of the theory of relativity: an experimental demonstration of the constancy of the velocity of light reflected by a moving mirror, Phys. Rev. 11, 411-420.

Majorana, Q. (1919), Experimental demonstration of the constancy of velocity of light emitted by a moving source, Phil. Mag. 37, 145-150.

Michelson, A. A. (1913), Effect of reflection from a moving mirror on the velocity of light. Astrophys. J. 37, 190-193.

Ritz, W. (1908), Récherches eritiques sur l'électrodynamique générale, Ann. Chim. et Phys. 13, 145-275.

Rotz, F. B. (1963), New test of the velocity of light postulate, Phys. Letters $\boldsymbol{\gamma}, 252-254$.

Tolman, R. C. (1910), The second postulate of relativity, Phys. Rev. 31, 26-40.

Tolman, R. C. (1912), Some emission theories of light, Phys. Rev. 35, 136-143.

Tomaschek, R. (1924), Über das Verhalten des Lichtes ausserirdischer Lichtquellen, Ann. d. Phys. 73, 105-126.

(Paper 68D12-430) 